OPEN ACCESS

Edited by:

Charles A. Kunos,

Investigational Drug Branch, National Cancer Institute $(\mathrm{NIH})$, United States

Reviewed by:

Sarah M. Temkin,

Virginia Commonwealth University, United States

Jyoti Mayadev,

University of California, San Diego,

United States

*Correspondence:

Ekaterina S. Jordanova

e.jordanova@vumc.nl

tThese authors have contributed equally to this work

Specialty section:

This article was submitted to

Women's Cancer

a section of the journal

Frontiers in Oncology

Received: 04 October 2018

Accepted: 10 January 2019

Published: 29 January 2019

Citation:

Sahasrabudhe NM, van der Horst JC, Spaans V, Kenter G, de Kroon C,

Bosse T, van Vliet SJ and Jordanova ES (2019) MGL Ligand

Expression Is Correlated to Lower

Survival and Distant Metastasis in

Cervical Squamous Cell and

Adenosquamous Carcinoma.

Front. Oncol. 9:29.

doi: 10.3389/fonc.2019.00029

\section{MGL Ligand Expression Is Correlated to Lower Survival and Distant Metastasis in Cervical Squamous Cell and Adenosquamous Carcinoma}

\author{
Neha M. Sahasrabudhe ${ }^{1}$, Joost C. van der Horst ${ }^{1}$, Vivian Spaans ${ }^{2,3}$, Gemma Kenter ${ }^{4}$, \\ Cor de Kroon ${ }^{3}$, Tjalling Bosse ${ }^{2}$, Sandra J. van Vliet ${ }^{1 \dagger}$ and Ekaterina S. Jordanova ${ }^{2,4 * \dagger}$ \\ ${ }^{1}$ Department of Molecular Cell Biology and Immunology, Cancer Center Amsterdam, Amsterdam Infection \& Immunity \\ Institute, Amsterdam UMC, Vrije Universiteit Amsterdam, Amsterdam, Netherlands, ${ }^{2}$ Department of Pathology, Leiden \\ University Medical Centre, Leiden, Netherlands, ${ }^{3}$ Department of Obstetrics and Gynecology, Leiden University Medical \\ Centre, Leiden, Netherlands, ${ }^{4}$ Department of Obstetrics and Gynecology, Center for Gynecological Oncology Amsterdam, \\ Amsterdam UMC, Vrije Universiteit Amsterdam, Amsterdam, Netherlands
}

Cervical cancer is the fourth most common cancer type in women worldwide and is characterized by a highly immune-suppressive microenvironment. Here, we describe aberrant glycosylation as a factor mediating this immunosuppressive microenvironment. Expression of a specific carbohydrate ligand for the immune-regulatory C-type lectin MGL was correlated to poor disease-specific survival and distant recurrences in squamous cell carcinoma (SCC) and adenosquamous carcinoma (ASC), the most common histological subtypes of cervical cancer. MGL ligand expression was also associated with lymph node metastasis, the absence of $\mathrm{CD}_{1} 4^{+}$myeloid cells and the presence of CD14- CD163 ${ }^{+}$ myeloid cells. Indeed, expression of the MGL receptor itself could be detected on $\mathrm{CD}_{163^{+}}$cells, suggesting that $\mathrm{MGL}^{+}$myeloid cells are able to interact locally with MGL ligand ${ }^{+}$tumor cells. Additionally, MGL ligand expression correlated to the occurrence of PIK3CA mutations, the most frequently observed oncogenic alteration in cervical cancer. In conclusion, we present prognostic value for MGL ligand expression in SCC/ASC patients, which further supports an immune evasive role for the C-type lectin MGL in the tumor immune compartment.

Keywords: MGL, glycosylation, cervical cancer, distant metastasis, histological subtype

\section{INTRODUCTION}

Cervical cancer is the fourth most common cancer in women worldwide (1). Histologically cervical cancer is subdivided into squamous cell carcinoma (SCC), adenocarcinoma (AC), and adenosquamous carcinoma (ASC), of which $75-80 \%$ are of SCC subtype and $15-20 \%$ of AC and ASC combined (2). Almost all SCC, AC, and ASC cervical cancer cases are due to persistent human papilloma virus (HPV) infection and gradually accumulating mutations (3). Although cervical cancer spreads in a predictable stage wise manner, disease recurrence is still a major concern. The primary treatment for stage IB-IIA cervical cancer is either radical surgical removal coupled with adjuvant chemo-radiotherapy or primary chemo-radiotherapy (4). After adjuvant radiotherapy recurrences are only reduced by approximately $50 \%$ (5), suggesting that new prognostic markers would be beneficial in predicting disease-free recurrence and therapeutic success. 
The development of cancer from the initial stage of HPV infection to the final invasive carcinoma depends on the immune response mounted by the host against HPV. Only $1 \%$ of women develop invasive carcinoma following the HPV infection due to an effective cellular immune response against cancerous cells (6). However, in patients having invasive carcinoma, the cancer is characterized by a highly immunosuppressive tumor microenvironment. Increased frequencies of regulatory $\mathrm{T}$ cells, and $\mathrm{T}$ cells expressing high levels of the immune checkpoint molecules PD-1 and CTLA-4 were observed in lymph nodes with metastasized cervical tumor compared to tumor negative lymph nodes (7). We and others, have reported on various factors mediating tumor immune suppression such as high expression of HLA- E coupled with lower infiltration of $\mathrm{CD}^{+}{ }^{+} \mathrm{T}$ cells and NK cells $(8,9)$, reduced HLA class I expression (10), reduced infiltration of M1 type macrophages (11), increased PDL-1 expression on both tumor cells and myeloid cells (7, 12), increased Indoleamine-2,3-dioxygenase (IDO) metabolic activity (13) and reduced major histocompatibility complex class I chain-related molecule A (MICA) expression (14) aiding immune escape by cervical cancer. Also, the different histological subtypes have different genomic and immunological characteristics $(9,14-17)$.

Aberrant glycosylation is a principal, yet underappreciated, factor mediating tumor immune escape (18). Glycosylation is the most abundant post-translational modification of proteins and lipids. However, during tumor progression an altered glycosylation pattern is observed in tumor cells compared to their non-transformed counterparts (19). Often, elevated expression of tumor-associated glycans is associated with increased aggressiveness, metastasis of the disease and hampered immune recognition of tumors (19). Epithelial cancers, such as cervical cancer, have a highly glycosylated surface and are associated with increased expression of truncated $O$-glycans such as Tn antigen (GalNAca-Ser/Thr) $(20,21)$. We have previously demonstrated that Tn antigen expressed on the mucin MUC1 is bound by the glycan-binding receptor macrophage galactosetype lectin (MGL/CLEC10A/CD301) (22). MGL is a C-type lectin receptor expressed specifically in myeloid cells, such immature tolerogenic dendritic cells and macrophages (23). The interaction of MGL with its ligand on effector T cells blocks $\mathrm{T}$ cell activation and induces $\mathrm{T}$ cell apoptosis (24). Furthermore, in colorectal cancer, high MGL ligand expression in tumors is associated with poor disease-free survival in late stage disease, whereby MGL ligand expression was highly correlated to mutations in the BRAF gene (25). While it is established that MGL ligand binding leads to an immune regulatory response (26), whether MGL binding has a role in mediating immune-evasion and tumor growth in cervical cancer patients has not been determined yet. In the present study we analyzed a cohort of cervical cancer samples for binding to chimeric MGL receptors and determined whether MGL binding was correlated to clinical and immune parameters for disease progression and aggressiveness of the tumor. Our results demonstrate a positive correlation between MGL binding and distant metastasis in cervical (adeno-)squamous cell carcinoma and a reduced recurrence-free survival.

\section{MATERIALS AND METHODS}

\section{Subjects and Follow-Up Data}

were retrieved from Department of Pathology, Leiden University Medical Center, Leiden, The Netherlands. From 109 patients with cervical carcinoma who underwent radical hysterectomy with bilateral pelvic lymphadenectomy between 1985 and 1999 , formalin-fixed, paraffin-embedded tissue blocks were retrieved from the archives. These cases were chosen on the basis of availability of sufficient material. All cases were used according to the Code of Conduct for Proper Secondary Use of Human Tissue, established by the Federation of Dutch Medical Scientific Societies (www.hedera.org) (27). Importantly, all these cases were previously subjected to thorough analyses by additional PAS +/AB staining to determine their exact histological subtype (17). Patients which were treated with radiotherapy and/or chemotherapy prior to surgery were excluded from the sample group. Follow-up data in this database was recorded from the date of primary surgery till September 2016. Follow-up data was recorded prospectively in a de-identified manner and was updated directly from medical records of patients. Followup time in months was between primary surgery and: (1) death caused by cervical cancer, while patients who died of a cause unrelated to cervical cancer are considered as censored observations at the date of death (disease-specific survival); and (2) distant metastasis or locoregional recurrence, whichever occurred first, or when both occurred within 30 days both were stated. Death was considered as a censored observation (diseasefree survival).

\section{Immunohistochemistry Using Tissue Microarrays}

Tissue microarray (TMA) were created by punching three core biopsies with a diameter of $0.6 \mathrm{~mm}$ from the central part of the tumor (28). Core biopsies were transfer to a recipient paraffin block. TMAs were sectioned in $4 \mu \mathrm{m}$ size, deparaffinized and rehydrated. Endogenous peroxidase was blocked using $0.03 \%$ hydrogen peroxide in $70 \%$ ethanol for $20 \mathrm{~min}$, followed by incubation with MGL-mouseFc for $2 \mathrm{~h}$ at RT (29). Then, slides were washed with PBS and incubated with Goat antimouse IgG Fc-PO (Jackson Immunoresresearch Laboratories) for $1 \mathrm{hr}$. Complexes were visualized using 3,3'-diaminobenzidine tetrahydrochloride (Sigma, USA). Slides were counterstained with Haematoxylin and coverslipped. MGL binding was scored in tumor fields and subsequently grouped in two groups containing negative/weak and positive/strong cases.

\section{Immunofluorescence}

Immunostaining was performed on $4 \mu \mathrm{m}$ thick formalin fixed paraffin embedded tumor sections using anti-MGL antibodies synthesized in our laboratory (IG6.6, IgG2a) (23) and antiCD163 antibody (NCL-CD163 LeicaBiosystems, IgG1). Antigen retrieval was performed using Tris-ethylenediaminetetraacetic acid (EDTA) buffer (10 mM Tris plus $1 \mathrm{mM}$ EDTA pH 9.0). Antigen retrieval was followed by overnight incubation at room temperature with anti-MGL and anti-CD163 antibody diluted in 

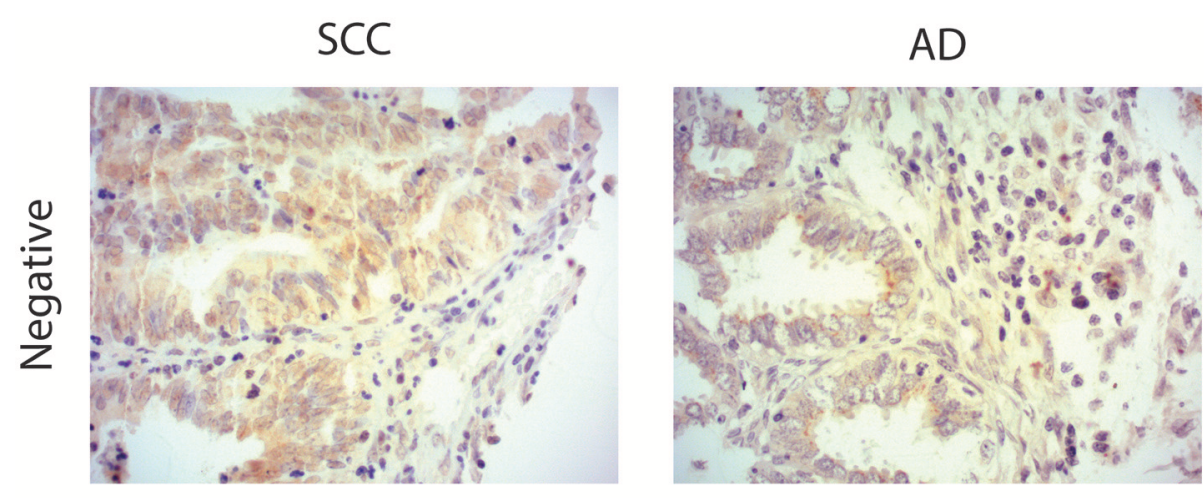

SCC

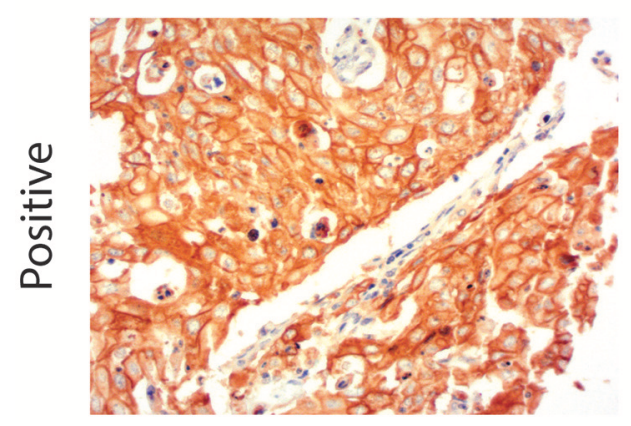

ASC

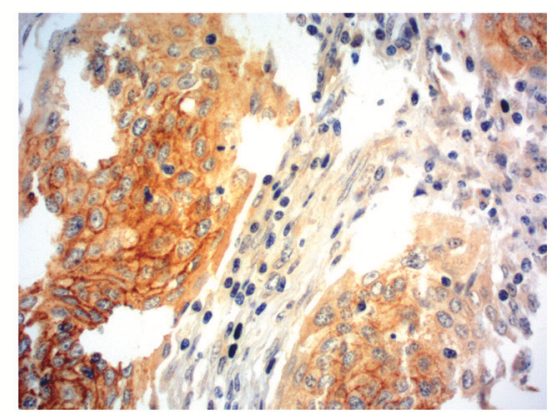

FIGURE 1 | MGL ligand expression in cervical cancer. Representative images of Squamous Cell Carcinoma (SCC), Adenosquamous carcinoma (ASC), and adenocarcinoma (AC) cervical cancer tissues stained with a chimeric MGL-mouseFc protein. MGL ligand expression was labeled positive or negative/weak based on the intensity of MGL-mouseFc binding. Magnification 20x.

$1 \% \mathrm{w} / \mathrm{v}$ bovine serum albumin in PBS. Alexa Fluor labeled Goatanti-mouseIgG1-A488 (CD163) and Goat-anti-mouseIgG2aA546 (both from Invitrogen, Life Technologies, Carlsbad, USA) were incubated at room temperature for 1 hour. Slides were mounted using VectaShield mounting medium containing DAPI. Images were obtained using an LSM700 confocal laser scanning microscope containing an LCI Plan-Neofluar 25×/0.8 Imm Korr DIC M27 objective (Zeiss, Göttingen, Germany).

\section{Somatic Mutation Genotyping}

Previously published data on somatic mutation genotyping using the GynCarta 2.0 mutation panel (Sequenom, Hamburg, Germany) was used (30). This panel analyzes mutations that are most commonly involved in gynecological malignancies, detecting 171 mutations in 13 genes: BRAF, CDKN2A, CTNNB1, FBXW7, FGFR2, FGFR3, FOXL2, HRAS, KRASNRAS, PIK3CA, $P P P 2 R 1 A$, and PTEN. The mutation spectra of these samples were published previously $(17,30)$.

\section{Statistics}

To determine association between MGL ligand expression and clinocopathologic characteristics, the chi-squared $\left(\chi^{2}\right)$ test was used. Univariate Cox-regression analysis was performed to assess the association with disease-free survival. Survival curves were estimated by the Kaplan-Meier method. Multivariate Cox-regression analysis was performed to determine whether
MGL ligand expression was independently associated with HPV type, FIGO disease staging, histopathological diagnosis, tumor size, infiltration depth, parametrial invasion, vaso-invasion, and lymph node metastasis. These factors were considered as covariates and included in multivariate analyses and if $p<0.10$ in univariate analysis. To determine the association between MGL ligand expression and oncogenic mutations chi-squared $\left(\chi^{2}\right)$ test was used. Significance tests were two-sided and statistical significance was assumed when $p<0.05$, corresponding to $95 \%$ confidence intervals (CI). Statistical analyses were performed using IBM SPSS Statistics 23. This study is reported according to Reporting recommendations for tumor MARKer prognostic studies (REMARK) (31).

\section{RESULTS}

\section{MGL Ligand Expression in SCC/ASC Correlates to Lymph Node Metastasis}

Due to the well-defined, highly immunosuppressive tumor microenvironment in cervical cancer, we investigated whether MGL ligand expression is also correlated to different clinical parameters of cervical cancer patients. MGL ligand expression in tumor samples was determined using a chimeric MGL-mouseFc protein, which detects the presence of MGL binding glycans in the tissue (Figure 1). Of the 109 patient samples included in the TMA, MGL staining could be analyzed in 96, others were 
TABLE 1 | Clinicopathologic characteristics of the studied cohort $(n=96)$ in relation to $\mathrm{MGL}$-ligand expression.

\begin{tabular}{|c|c|c|c|c|}
\hline & Total & MGL-ligand weak & $\begin{array}{l}\text { MGL-ligand } \\
\text { strong }\end{array}$ & \\
\hline & $N(\%)$ & $N(\%)$ & $N(\%)$ & P-value \\
\hline \multicolumn{5}{|c|}{ HPV TYPE } \\
\hline Negative & $7(7.3)$ & $5(71.4)$ & $2(28.6)$ & \\
\hline 16 & $50(52.1)$ & $35(70)$ & $15(30)$ & \\
\hline 18 & $20(20.8)$ & $14(70)$ & $6(30)$ & \\
\hline Other & $16(16.7)$ & $10(62.5)$ & $6(37.5)$ & 0.1 \\
\hline Unknown & $3(3.1)$ & & & \\
\hline \multicolumn{5}{|l|}{ FIGO } \\
\hline IB1 & 45 (46.9) & $29(64.4)$ & $16(35.6)$ & \\
\hline IB2 & $31(32.3)$ & $22(71)$ & $9(29)$ & \\
\hline$\| \mathrm{A}$ & $20(20.8)$ & $14(70)$ & $6(30)$ & 0.8 \\
\hline \multicolumn{5}{|c|}{ HISTOPATHOLOGY } \\
\hline SCC & $59(61.5)$ & $34(57.6)$ & $25(42.4)$ & \\
\hline ASC & $23(24)$ & 18 (78.3) & $5(21.7)$ & \\
\hline$A C$ & $14(14.6)$ & $13(92.9)$ & $1(7.1)$ & 0.02 \\
\hline \multicolumn{5}{|c|}{ TUMOR SIZE } \\
\hline$<40 \mathrm{~mm}$ & $54(56.3)$ & $38(70.4)$ & $16(29.6)$ & \\
\hline$\geq 40 \mathrm{~mm}$ & $37(38.5)$ & $22(59.5)$ & $15(40.5)$ & \\
\hline Unknown & $5(5.2)$ & & & 0.3 \\
\hline \multicolumn{5}{|c|}{ INFILTRATION DEPTH } \\
\hline$<15 \mathrm{~mm}$ & $51(53.1)$ & $38(74.5)$ & $13(25.5)$ & \\
\hline$\geq 15 \mathrm{~mm}$ & 45 (46.9) & $27(60)$ & $18(40)$ & 0.1 \\
\hline \multicolumn{5}{|c|}{ PARAMETRIAL INVASION } \\
\hline Negative & $86(89.6)$ & $60(69.8)$ & $26(30.2)$ & \\
\hline Positive & $10(10.4)$ & $5(50)$ & $5(50)$ & 0.2 \\
\hline \multicolumn{5}{|c|}{ VASOINVASION } \\
\hline Negative & $45(46.9)$ & 33 (73.3) & $12(26.7)$ & \\
\hline Positive & $48(50)$ & $29(60.4)$ & $19(39.6)$ & 0.2 \\
\hline Unknown & $3(3.1)$ & & & \\
\hline \multicolumn{5}{|c|}{ LYMPH NODE METASTASIS } \\
\hline Negative & $70(72.9)$ & $51(72.9)$ & $19(27.1)$ & \\
\hline Positive & $26(27.1)$ & $14(53.8)$ & $12(46.2)$ & $0.07\left(0.04^{\star}\right)$ \\
\hline \multicolumn{5}{|c|}{ POSTOPERATIVE RADIOTHERAPY } \\
\hline Yes & $54(56.3)$ & 32 (59.3) & $22(40.7)$ & 0.05 \\
\hline No & $42(43.7)$ & $33(78.6)$ & $9(21.4)$ & \\
\hline
\end{tabular}

P-value obtained with the Chi-square test. ${ }^{*}$ Statistical values in SCC/ASC patient subgroup. HPV, human papillomavirus; FIGO, international federation of gynecology and Obstetrics stage for cervical carcinoma; SCC, squamous cell carcinoma; ASC, adenosquamous carcinoma; AC, adenocarcinoma. Bold values signify significance.

excluded due to lack of tumor cells or loss of tumor core material. Patients were segregated based on clinicopathological parameters such as HPV subtype, International federation of Gynecology and Obstetrics (FIGO) staging, histopathology, tumor size, tumor infiltration depth, parametrial invasion, vaso-invasion, and lymph node metastasis (Table 1). Among all the parameters analyzed, a significant positive correlation between MGL ligand expression and histopathological subgroups was determined ( $p$ $=0.02$ ), whereby high MGL ligand expression was observed in $42.4 \%$ of the SCC samples and $21.7 \%$ of the ASC. In contrast, only
$7 \%$ of the AC tumors displayed high MGL ligand expression. As MGL ligand expression was mainly restricted to SCC/ASC group of patients, we continued our analysis using these two histological subtypes. We next compared all clinicopathological parameters to the SCC/ASC tumors and observed a significant correlation between MGL ligand expression and a higher frequency of lymph node metastasis $(p=0.04)$.

\section{MGL Ligand Expression in SCC/ASC Correlates to Reduced Distant Recurrence-Free Survival}

As MGL binding was related to a higher frequency of lymph node metastasis, we also determined whether high MGL binding was associated with a decreased disease-specific survival of patients within the SCC/ASC group. Lower disease-specific survival was found in the high MGL ligand group ( $p=0.044$; Figure 2A). In addition, when we differentiated to recurrence-free survival based on locoregional recurrence and distant recurrence, we observed clear differences between the high and low MGL ligand expressing patient groups. While no statistically significant difference in locoregional recurrence was observed (Figure 2B), MGL ligand expression was significantly correlated to distant recurrences ( $p=0.004$; Figure 2C).

\section{MGL Receptor Is Expressed on Myeloid Cells in Cervical Cancer Patients}

We have determined MGL ligand expression in tumor samples from cervical cancer patients. The clinical outcome in patients having MGL ligand expression, however depends equally on expression of MGL receptor in the tumor. Myeloid cell infiltration data, comprising subpopulations expressing CD14, CD33, and CD163 was previously described for this cohort and was integrated in the present study to determine association of MGL ligand expression with myeloid cell subpopulations (11). Using the non-parametric Mann-Whitney test, a positive correlation between $\mathrm{CD} 163^{+} \mathrm{CD} 14^{-}$myeloid cells and high MGL ligand expression ( $p=0.029$ ) was observed, while there were

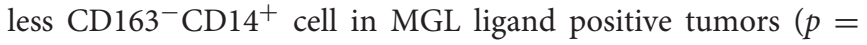
0.012). We next assessed by double immunofluorescent staining on the same TMA with an anti-MGL and CD163 antibodies, if the MGL receptor was expressed on the same $\mathrm{CD} 163^{+} \mathrm{CD} 14^{-}$ myeloid cells (Figure 3). Indeed, the MGL receptor was highly expressed in the stroma. Furthermore, MGL co-localization could be observed on a subset of the $\mathrm{CD} 163^{+}$tumor-associated myeloid cells (Figure 3).

\section{MGL Ligand Expression in SCC/ASC Correlates to PIK3CA Mutations}

We have previously demonstrated that MGL ligand expression is associated with BRAF mutations in colorectal cancer (25). Thus, we sought for possible associations between MGL ligand expression and known somatic mutations in cervical cancer tumors (30). The combined somatic mutation analysis in $B R A F$, CDKN2A, CTNNB1, FBXW7, FGFR2, FGFR3, FOXL2, HRAS, KRAS, NRAS, PIK3CA, PPP2R1A, and PTEN genes showed significant correlation to MGL ligand expression $(p=0.027)$, 

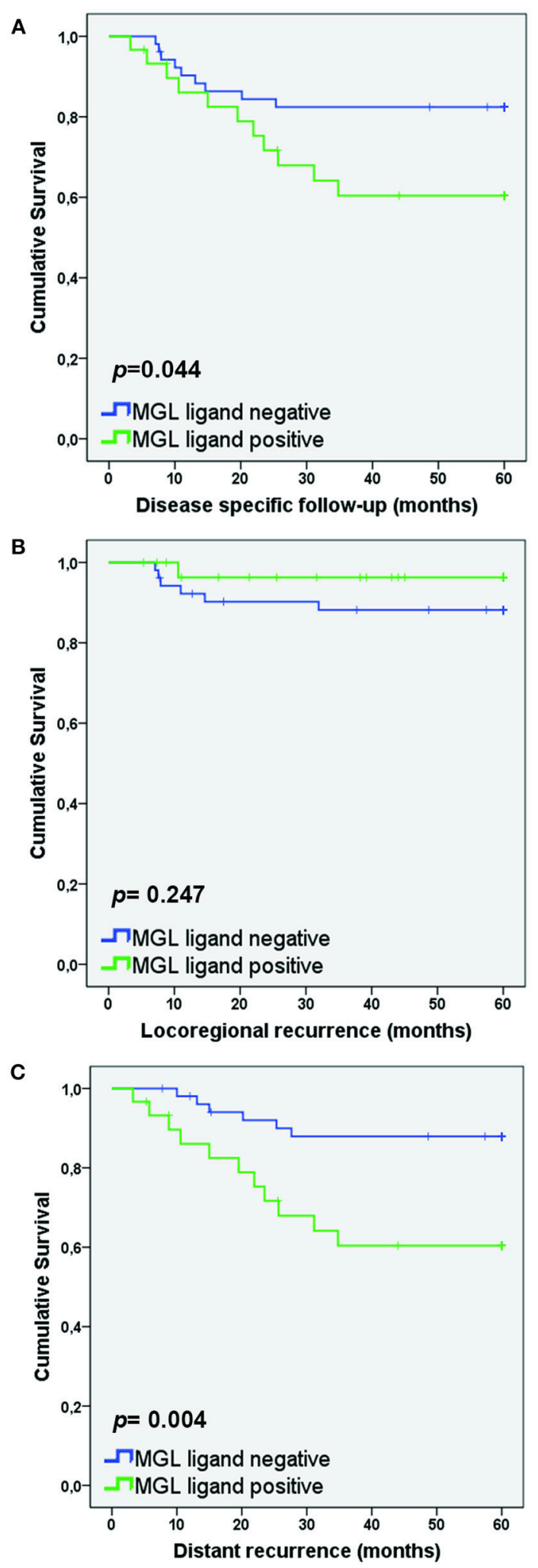

FIGURE 2 | MGL ligand expression correlates to lower survival in patients with distant recurrence. Kaplan Meier survival curves were plotted for SCC/AC patients for Disease-specific survival (A), Locoregional recurrence (B), and Distant recurrence (C). P-values determined by Log rank test. however this correlation was only marginal (Table 2). After individual analysis of mutations in BRAF, CDKN2A, CTNNB1, FBXW7, FGFR2, FGFR3, FOXL2, HRAS, KRASNRAS, PIK3CA, PPP2R1A, and PTEN genes with respect to MGL ligand expression, we only observed a significant correlation only to E542K and E545K mutations in PI3K ( $p=0.006$; Table 2).

\section{DISCUSSION}

Aberrant glycosylation in the tumor microenvironment is recognized as one of the principal factors mediating aggressive tumor growth (32). Certain glycan structures have a pivotal role in mediating immune suppression in tumor microenvironment. Binding of tumor associated glycan ligands such as the Tn antigen to the MGL receptor is one such mechanism by which tumors succeed in immune evasion accompanied by the aggressive tumor phenotype and lower recurrence-free survival (19, 24, 25). Here we determined that in (adeno-)squamous cervical cancer, high levels of MGL ligand expression correlated to lymph node metastasis and reduced distant recurrence-free survival. Thus, similar to colorectal cancer, also in cervical cancer MGL ligand expression was associated with a more aggressive tumor phenotype (25). Concomitant with the high levels of MGL ligands, MGL receptor expression was observed in myeloid cells in tumor stroma, more specifically in $\mathrm{CD}_{163}{ }^{+}$myeloid cells. Furthermore, the association between MGL ligand expression and oncogenic PIK3CA mutations suggest a causative role of PIK3CA mutation in MGL ligand expression in cervical cancer. Thus, here we elaborated on the role of MGL ligand expression and aberrant glycosylation in cervical cancer progression.

In our analysis, MGL ligand expression was mainly observed in SCC/ASC and not in the AC histological subtype. Among the histological subtypes of cervical cancer, SCC is the most predominant histological subtype followed by ASC/AC accounting for about $10-15 \%$ of total patients $(33,34)$. Apart from the histological differences between the subgroups, AC differs from both SCC and ASC with respect to metastasis, pattern of tumor growth, therapeutic regime as well as oncogenic mutations $(17,35-37)$. Analyses from our previous studies have demonstrated a significant difference between $\mathrm{AC}$ and SCC/ASC with respect to frequencies of an immunosuppressive microenvironment, where AC show improved survival with high immunosuppressive environments, while SCC/ASC show the opposite $(9,14,16)$. The non-classical HLA molecule HLAE is weakly expressed in healthy cervical epithelium while its expression increases with progression to higher stages of cancer $(8,38)$. HLA-E is proven to be a mediator for immune escape in virally infected cells and tumor cells $(8,39,40)$. Previously, we have shown that HLA-E expression is significantly higher in the AC subgroup than in SCC/ASC patients and high HLAE expression was correlated to improved disease-specific and recurrence-free survival (9). Complete loss of classical HLA class I molecules is observed more on SCC than in AC, again a characteristic of a highly immunosuppressive microenvironment $(10,41)$. In the same line of reasoning, a higher frequency of Tregs was correlated to better survival in AC (16) but poorer survival in 

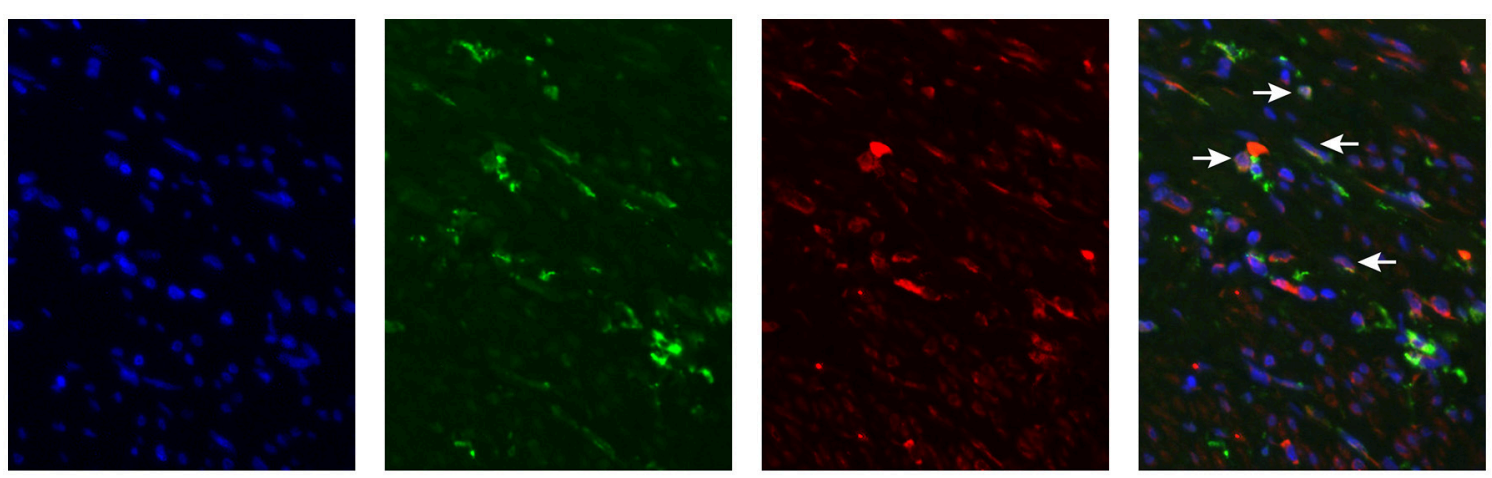

FIGURE 3 | MGL expression in Cervical Squamous cell carcinoma. MGL receptor (red), CD163 (green, myeloid cells) staining in formalin fixed tumor tissue. Nuclei were stained with DAPI (in blue).

TABLE 2 | MGL ligand expression in relation to mutational status.

\begin{tabular}{lcccc}
\hline & Total & $\begin{array}{c}\text { Weak MGL-ligand } \\
\text { expressions }\end{array}$ & $\begin{array}{c}\text { Strong MGL-ligand } \\
\text { expression }\end{array}$ & \\
\cline { 2 - 3 } & $\boldsymbol{N}^{\mathbf{a}(\%)}$ & $\mathbf{N}(\%)$ & $\mathbf{N}(\%)$ & P-value \\
\hline SOMATIC MUTATIONS & & & \\
No & $56(68.3)$ & $40(71.4)$ & $16(28.6)$ & \\
Yes & $26(31.7)$ & $12(46.1)$ & $14(53.8)$ & \\
PI3K MUTATIONS & & & \\
No & $63(76.8)$ & $45(71.4)$ & $18(28.6)$ & \\
Yes & $19(23.2)$ & $7(36.8)$ & $12(63.2)$ & 0.006 \\
\hline
\end{tabular}

${ }^{a}$ Total number of cases $=82$.

$P$-value obtained with the Chi-square test.

SCC $(42,43)$. Thus, an immunosuppressive microenvironment specifically results in poor survival in SCC patients, in contrast to patients with AC. This contrasting role of the immune microenvironment was also observed in the present study in relation to MGL ligand expression. We observed MGL ligand expression mainly in SCC/ASC groups, while minimal expression was observed in AC. MGL ligand expression in SCC/ASC also correlated to lymph node metastasis, reduced disease-specific survival and reduced distant recurrence-free survival. Thus, we propose that expression of MGL ligands in SSC/ASC is one of the factors mediating immune suppression which is correlated to poor survival, while the same mechanism might not apply to patients in the AC subgroup. These results further corroborate the differences between histological subtypes of cervical cancer and the need for opposing prognostic markers and therapeutic strategies.

Persistent carcinogenic HPV infection is the causative agent in $95 \%$ of cervical cancer patients. Following HPV infection, disease progression is frequently accompanied with genomic instability and accumulation of somatic mutations (15). The mutational profile of cervical cancers is a factor determining disease outcome as well as the histological subtype differentiation $(15,17)$. Among the somatic mutations observed, activating mutations in the PIK3CA gene, encoding the catalytic subunit of PI3K, are the most frequently observed mutations accounting for $20-36 \%$ of cervical cancer tumors $(17,37,44-46)$. The PI3K-AKT-mTOR pathway controls several aspects of tumor growth such as avoiding cell death, metabolism, metastasis and angiogenesis (47). PI3K activation has been implicated in glycosylation pathways before. In bladder cancer, the PI3KAKT-mTOR pathway was associated with sialyl-Tn antigen, whereby inhibition of mTOR led to reduced expression of this sialyl-Tn antigen (48). Additionally, expression of $\beta 1,6 \mathrm{GlcNAc-}$ branched $\mathrm{N}$-glycans on $\beta 4$ integrin is regulated by the PI3K pathway. Galectin-3 binding to $\beta 1,6$ GlcNAc-branched Nglycans on $\beta 4$ integrin mediates tumor growth and progression (49). Here, we observed a positive correlation between PI3K mutations and MGL ligand expression. With respect to tumorassociated glycosylation, we have determined that in colorectal cancer, BRAF mutations drive the expression of MGL ligands such as Tn antigen $(25,50)$. Intriguingly, mutations in the MAPK pathway also constitutively activate the PI3K pathway (47), suggesting that the PI3K-AKT-mTOR pathway could be responsible for enhancing MGL ligand expression. However, further investigation is needed for confirm this causative mechanistic link.

Myeloid cells in the tumor microenvironment are critical factors determining the aggressive nature of a tumor (51). Frequency and polarization of tumor-infiltrating myeloid cells are correlated to disease progression and disease-free survival. $\mathrm{CD}_{14}{ }^{+} \mathrm{CD}_{3}{ }^{-} \mathrm{CD} 63^{-}$myeloid cells are correlated to high frequencies of cytotoxic $\mathrm{T}$ cells and higher influx of both these cell types together is associated with improved diseasespecific survival (11). Interestingly, here we found an association of high numbers of this $\mathrm{CD}^{+} 4^{+}$subpopulation with low MGL ligand expression. In addition, we observed a positive correlation between $\mathrm{CD}_{14}{ }^{-} \mathrm{CD} 163^{+}$myeloid cells and MGL ligand expression. Moreover, MGL receptor expression could be visualized on $\mathrm{CD}_{163}{ }^{+}$cells. The MGL receptor is known to be expressed on myeloid cells such as macrophages and dendritic cells, where engagement of MGL ligands with MGL has been described to induce immune tolerance $(26,52)$. CD14 ${ }^{-} \mathrm{CD} 163^{+}$ myeloid cells, which we observed to be correlated to high MGL ligand expression have previously been correlated to a high 
frequency of $\mathrm{CD}^{+} \mathrm{CD}^{-}{ }^{-}$Foxp $3^{+}$regulatory T cells (11). Thus, our results are in coherence with the known immune-suppressive mechanisms of MGL triggering.

The here described cohort has several limitations; there is a limited number of adenocarcinoma patients and more than half of the patients included received post-surgical treatment radiotherapy. Although this did not have an effect on the role of MGL expression in SCC/ASC survival, a confirmation of our findings in an independent cohort of early stage cervical cancer patients is needed. Moreover, we were not able to study MGL expression in advanced disease as these patients receive chemotherapy and no surgical treatment.

Cervical cancer progression follows a predictable pattern starting with a persistent oncogenic HPV infection, followed by uncontrolled proliferation, eventually leading to metastasis to local lymph nodes and finally distant metastasis (53). Five year survival rates of patients with distant metastasis is drastically reduced to $16.5 \%$ compared to localized cervical cancer at $91.5 \%$ (54). Novel therapeutic regimes to tackle this dismal survival rate in late stage patients involve immunotherapy against the cancer (55). Aberrant glycosylation is increasingly recognized as factor mediating immune escape in the tumor microenvironment

\section{REFERENCES}

1. Ferlay J, Soerjomataram I, Dikshit R, Eser S, Mathers C, Rebelo M, et al. Cancer incidence and mortality worldwide: sources, methods and major patterns in GLOBOCAN 2012. Int J Cancer (2015) 136:E359-E86. doi: 10.1002/ijc.29210

2. Meijer CJLM, Steenbergen RDM. Gynaecological cancer: novel molecular subtypes of cervical cancer - potential clinical consequences. Nat Rev Clin Oncol. (2017) 14:397-8. doi: 10.1038/nrclinonc.2017.52

3. Schiffman M, Wentzensen N, Wacholder S, Kinney W, Gage JC, Castle PE. Human papillomavirus testing in the prevention of cervical cancer. JNCI: J Natl Cancer Inst. (2011) 103:368-83. doi: 10.1093/jnci/djq562

4. Sedlis A, Bundy BN, Rotman MZ, Lentz SS, Muderspach LI, Zaino RJ. A randomized trial of pelvic radiation therapy versus no further therapy in selected patients with stage IB carcinoma of the cervix after radical hysterectomy and pelvic lymphadenectomy: a gynecologic oncology group study. Gynecol Oncol. (1999) 73:177-83. doi: 10.1006/gyno.1999.5387

5. Rotman M, Sedlis A, Piedmonte MR, Bundy B, Lentz SS, Muderspach LI, et al. A phase III randomized trial of postoperative pelvic irradiation in stage IB cervical carcinoma with poor prognostic features: follow-up of a gynecologic oncology group study. Int J Radiation Oncol Biol Phys. (2006) 65:169-76. doi: 10.1016/j.ijrobp.2005.10.019

6. Tindle RW. OPINIONImmune evasion in human papillomavirus-associated cervical cancer. Nat Rev Cancer (2002) 2:59-64. doi: 10.1038/nrc700

7. Heeren AM, Koster BD, Samuels S, Ferns DM, Chondronasiou D, Kenter GG, et al. High and interrelated rates of PD-L1+CD14+ antigen-presenting cells and regulatory $\mathrm{T}$ cells mark the microenvironment of metastatic lymph nodes from patients with cervical cancer. Cancer Immunol Res. (2014) 3:48-58. doi: 10.1158/2326-6066.cir-14-0149

8. Gooden M, Lampen M, Jordanova ES, Leffers N, Trimbos JB, van der Burg SH, et al. HLA-E expression by gynecological cancers restrains tumorinfiltrating CD8+ T lymphocytes. Proc Natl Acad Sci USA. (2011) 108:1065661. doi: 10.1073/pnas.1100354108

9. Spaans VM, Peters AAW, Fleuren G, Jordanova ES. HLA-E expression in cervical adenocarcinomas: association with improved long-term survival. $J$ Transl Med. (2012) 10:184. doi: 10.1186/1479-5876-10-184

10. Ferns DM, Heeren AM, Samuels S, Bleeker MCG, de Gruijl TD, Kenter GG, et al. Classical and non-classical HLA class I aberrations in primary and blocking these immunosuppressive glycans is being explored as a new therapeutic option (56). In this article we have determined that MGL ligand expression is linked to both lymph node and distant metastasis in squamous cervical tumors. Additionally, MGL ligand expression is correlated to PIK3CA mutations, the most frequent oncogenic mutation in cervical cancer. Thus, MGL ligand expression may be utilized as a new prognostic and therapy outcome marker. Additionally, blocking of MGL could present as a novel therapeutic option for improved immune eradication of (adeno-) squamous cervical tumors.

\section{AUTHOR CONTRIBUTIONS}

NS assembled data and wrote the article. JvdH performed data confirmation. VS, GK, CdK, and TB were involved in collecting the data. SvV and EJ did study design and final review of manuscript. All authors have approved the final manuscript.

\section{FUNDING}

This work was supported by a grant from the Dutch Cancer Society (KWF, grant number VU 2014-6779).

cervical squamous- and adenocarcinomas and paired lymph node metastases. J Immunother Cancer (2016) 4:78. doi: 10.1186/s40425-016-0184-3

11. de Vos van Steenwijk PJ, Ramwadhdoebe TH, Goedemans R, Doorduijn EM, van Ham JJ, Gorter A, et al. Tumor-infiltrating CD14-positive myeloid cells and CD8-positive T-cells prolong survival in patients with cervical carcinoma. Int J Cancer (2013) 133:2884-94. doi: 10.1002/ijc.28309

12. Heeren AM, Punt S, Bleeker MC, Gaarenstroom KN, van der Velden J, Kenter GG, et al. Prognostic effect of different PD-L1 expression patterns in squamous cell carcinoma and adenocarcinoma of the cervix. Mod Pathol. (2016) 29:753-63. doi: 10.1038/modpathol.2016.64

13. Ferns DM, Kema IP, Buist MR, Nijman HW, Kenter GG, Jordanova ES. Indoleamine-2,3-dioxygenase (IDO) metabolic activity is detrimental for cervical cancer patient survival. Oncoimmunology (2015) 4:e981457. doi: 10.4161/2162402X.2014.981457

14. Samuels S, Ferns DM, Meijer D, van Straalen JP, Buist MR, Zijlmans HJ, et al. High levels of soluble MICA are significantly related to increased disease-free and disease-specific survival in patients with cervical adenocarcinoma. Tissue Antigens (2015) 85:476-83. doi: 10.1111/tan.12562

15. Burk RD, Chen Z, Saller C, Tarvin K, Carvalho AL, Scapulatempo-Neto C, et al. Integrated genomic and molecular characterization of cervical cancer. Nature (2017) 543:378-84. doi: 10.1038/nature21386

16. Punt S, van Vliet ME, Spaans VM, de Kroon CD, Fleuren GJ, Gorter A, et al. FoxP3+ and IL-17+ cells are correlated with improved prognosis in cervical adenocarcinoma. Cancer Immunol Immunother. (2015) 64:745-53. doi: 10.1007/s00262-015-1678-4

17. Spaans VM, Trietsch MD, Peters AA, Osse M, Ter Haar N, Fleuren GJ, et al. Precise classification of cervical carcinomas combined with somatic mutation profiling contributes to predicting disease outcome. PLoS ONE (2015) 10:e0133670. doi: 10.1371/journal.pone.0133670

18. Cornelissen LA, Van Vliet SJ. A bitter sweet symphony: immune responses to altered O-glycan epitopes in cancer. Biomolecules (2016) 6:E26. doi: 10.3390/biom6020026

19. Pinho SS, Reis CA. Glycosylation in cancer: mechanisms and clinical implications. Nat Rev Cancer (2015) 15:540-55. doi: 10.1038/nrc3982

20. Hirao T, Sakamoto Y, Kamada M, Hamada S-I, Aono T. Tn antigen, a marker of potential for metastasis of uterine cervix cancer cells. Cancer (1993) 72:154-9. doi: 10.1002/1097-0142(19930701)72:1<154::aid-cncr2820720129>3.0.co;2-c 
21. Hamada S, Furumoto H, Kamada M, Hirao T, Aono T. High expression rate of Tn antigen in metastatic lesions of uterine cervical cancers. Cancer Lett. (1993) 74:167-73.

22. Saeland E, van Vliet SJ, Bäckström M, van den Berg VCM, Geijtenbeek TBH, Meijer GA, et al. The C-type lectin MGL expressed by dendritic cells detects glycan changes on MUC1 in colon carcinoma. Cancer Immunol Immunother. (2006) 56:1225-36. doi: 10.1007/s00262-006-0274-Z

23. van Vliet SJ, van Liempt E, Geijtenbeek TBH, van Kooyk Y. Differential regulation of C-type lectin expression on tolerogenic dendritic cell subsets. Immunobiology (2006) 211:577-85. doi: 10.1016/j.imbio.2006.05.022

24. van Vliet SJ, Gringhuis SI, Geijtenbeek TBH, van Kooyk Y. Regulation of effector T cells by antigen-presenting cells via interaction of the C-type lectin MGL with CD45. Nat Immunol. (2006) 7:1200-8. doi: 10.1038/ni1390

25. Lenos K, Goos JACM, Vuist IM, den Uil SH, Delis-van Diemen PM, Belt EJT, et al. MGL ligand expression is correlated to BRAF mutation and associated with poor survival of stage III colon cancer patients. Oncotarget (2015) 6:26278-90. doi: 10.18632/oncotarget.4495

26. Li D, Romain G, Flamar A-L, Duluc D, Dullaers M, Li X-H, et al. Targeting self- and foreign antigens to dendritic cells via DC-ASGPR generates IL10-producing suppressive CD4+T cells. J Exp Med. (2012) 209:109-21. doi: $10.1084 /$ jem.20110399

27. Oosterhuis JW, Coebergh JW, van Veen EB. Tumour banks: well-guarded treasures in the interest of patients. Nat Rev Cancer (2003) 3:73-7. doi: $10.1038 / \mathrm{nrc} 973$

28. Mehta AM, Jordanova ES, Kenter GG, Ferrone S, Fleuren G-J. Association of antigen processing machinery and HLA class I defects with clinicopathological outcome in cervical carcinoma. Cancer Immunol Immunother. (2007) 57:197-206. doi: 10.1007/s00262-007-0362-8

29. van Vliet SJ, Paessens LC, Broks-van den Berg VCM, Geijtenbeek $\mathrm{TBH}$, van Kooyk Y. The C-type lectin macrophage galactose-type lectin impedes migration of immature APCs. J Immunol. (2008) 181:3148-55. doi: 10.4049/jimmunol.181.5.3148

30. Castresana JS, Spaans VM, Trietsch MD, Crobach S, Stelloo E, Kremer $\mathrm{D}$, et al. Designing a high-throughput somatic mutation profiling panel specifically for gynaecological cancers. PLoS ONE (2014) 9:e93451. doi: 10.1371/journal.pone.0093451

31. McShane LM, Altman DG, Sauerbrei W, Taube SE, Gion M, Clark GM. Reporting recommendations for tumor marker prognostic studies (REMARK). JNCI: J Natl Cancer Inst. (2005) 97:1180-4. doi: 10.1093/jnci/dji237

32. Munkley J, Elliott DJ. Hallmarks of glycosylation in cancer. Oncotarget (2016) 7:35478-89. doi: 10.18632/oncotarget.8155

33. Vizcaino AP, Moreno V, Bosch FX, Munoz N, Barros-Dios XM, Parkin DM. International trends in the incidence of cervical cancer: I. Adenocarcinoma and adenosquamous cell carcinomas. Int J Cancer (1998) 75:536-45.

34. Vizcaino AP, Moreno V, Bosch FX, Muñoz N, Barros-Dios XM, Borras J, et al. International trends in incidence of cervical cancer: II. Squamouscell carcinoma. Int J Cancer (2000) 86:429-35. doi: 10.1002/(SICI)10970215(20000501)86:3<429::AID-IJC20>3.0.CO;2-D

35. Gien LT, Beauchemin M-C, Thomas G. Adenocarcinoma: a unique cervical cancer. Gynecol Oncol. (2010) 116:140-6. doi: 10.1016/j.ygyno.2009.09.040

36. Rose PG. Are the differences in treatment outcome for adenocarcinoma of the cervix different enough to change the treatment paradigm? Gynecol Oncol. (2012) 125:285-6. doi: 10.1016/j.ygyno.2012.03.010

37. Wright AA, Howitt BE, Myers AP, Dahlberg SE, Palescandolo E, Van Hummelen $\mathrm{P}$, et al. Oncogenic mutations in cervical cancer: genomic differences between adenocarcinomas and squamous cell carcinomas of the cervix. Cancer (2013) 119:3776-83. doi: 10.1002/cncr.28288

38. Gonçalves MAG, Le Discorde M, Simões RT, Rabreau M, Soares EG, Donadi EA, et al. Classical and non-classical HLA molecules and p16INK4a expression in precursors lesions and invasive cervical cancer. Eur J Obstetr Gynecol Reproduct Biol. (2008) 141:70-4. doi: 10.1016/j.ejogrb.2008.06.010

39. Rodgers JR, Cook RG. MHC class Ib molecules bridge innate and acquired immunity. Nat Rev Immunol. (2005) 5:459-71. doi: 10.1038/nri1635

40. Cohen GB, Gandhi RT, Davis DM, Mandelboim O, Chen BK, Strominger JL, et al. The selective downregulation of class I major histocompatibility complex proteins by HIV-1 protects HIV-infected cells from NK cells. Immunity (1999) 10:661-71. doi: 10.1016/s1074-7613(00)80065-5
41. Hilders CG, Munoz IM, Nooyen Y, Fleuren GJ. Altered HLA expression by metastatic cervical carcinoma cells as a factor in impaired immune surveillance. Gynecol Oncol. (1995) 57:366-75. doi: 10.1006/gyno.1995.1156

42. Jordanova ES, Gorter A, Ayachi O, Prins F, Durrant LG, Kenter GG, et al. Human leukocyte antigen class I, MHC class I chain-related molecule $\mathrm{A}$, and $\mathrm{CD} 8+/$ regulatory $\mathrm{T}$-cell ratio: which variable determines survival of cervical cancer patients? Clin Cancer Res. (2008) 14:2028-35. doi: 10.1158/1078-0432.ccr-07-4554

43. Shah W, Yan X, Jing L, Zhou Y, Chen H, Wang Y. A reversed CD4/CD8 ratio of tumor-infiltrating lymphocytes and a high percentage of CD4+FOXP3+ regulatory $\mathrm{T}$ cells are significantly associated with clinical outcome in squamous cell carcinoma of the cervix. Cell Mol Immunol. (2010) 8:59-66. doi: $10.1038 / \mathrm{cmi} .2010 .56$

44. Janku F, Lee JJ, Tsimberidou AM, Hong DS, Naing A, Falchook GS, et al. PIK3CA mutations frequently coexist with RAS and BRAF mutations in patients with advanced cancers. PLOS ONE (2011) 6:e22769. doi: 10.1371/journal.pone.0022769

45. McIntyre JB, Wu JS, Craighead PS, Phan T, Kobel M, Lees-Miller SP, et al. PIK3CA mutational status and overall survival in patients with cervical cancer treated with radical chemoradiotherapy. Gynecol Oncol. (2013) 128:409-14. doi: 10.1016/j.ygyno.2012.12.019

46. Spaans VM, Nyoman Bayu Mahendra I, Purwoto G, Trietsch MD, Osse $\mathrm{M}$, Ter Haar $\mathrm{N}$, et al. The landscape of somatic mutations in Indonesian cervical cancer is predominated by the PI3K pathway. Gynecol Oncol. (2018) 148:189-96. doi: 10.1016/j.ygyno.2017.10.009

47. Fruman DA, Rommel C. PI3K and cancer: lessons, challenges and opportunities. Nat Rev Drug Discov. (2014) 13:140-56. doi: 10.1038/nrd4204

48. Costa C, Pereira S, Lima L, Peixoto A, Fernandes E, Neves D, et al. Abnormal protein glycosylation and activated PI3K/Akt/mTOR Pathway: role in bladder cancer prognosis and targeted therapeutics. PLoS ONE (2015) 10:e0141253. doi: 10.1371/journal.pone.0141253

49. Kariya Y, Oyama M, Hashimoto Y, Gu J, Kariya Y. beta4Integrin/PI3K signaling promotes tumor progression through the galectin-3-N-glycan complex. Mol Cancer Res. (2018) 16:1024-34 doi: 10.1158/1541-7786.MCR-17-0365

50. Sahasrabudhe NM, Lenos K, van der Horst JC, Rodriguez E, van Vliet SJ. Oncogenic BRAFV600E drives expression of MGL ligands in the colorectal cancer cell line HT29 through N-acetylgalactosamine-transferase 3. Biol Chem. (2018) 399:649-59. doi: 10.1515/hsz-2018-0120

51. Engblom C, Pfirschke C, Pittet MJ. The role of myeloid cells in cancer therapies. Nat Rev Cancer (2016) 16:447-62. doi: 10.1038/nrc. 2016.54

52. van Vliet SJ, Bay S, Vuist IM, Kalay H, García-Vallejo JJ, Leclerc C, et al. MGL signaling augments TLR2-mediated responses for enhanced IL-10 and TNF- $\alpha$ secretion. J Leukoc Biol. (2013) 94:315-23. doi: 10.1189/jlb.1012520

53. Haie-Meder C, Morice P, Castiglione M, Group EGW. Cervical cancer: ESMO clinical practice guidelines for diagnosis, treatment and follow-up. Ann Oncol. (2010) 21(Suppl. 5):v37-40. doi: 10.1093/annonc/mdq162

54. Li $\mathrm{H}, \mathrm{Wu} \mathrm{X}$, Cheng $\mathrm{X}$. Advances in diagnosis and treatment of metastatic cervical cancer. J Gynecol Oncol. (2016) 27:e43. doi: 10.3802/jgo.2016.27.e43

55. Orbegoso C, Murali K, Banerjee S. The current status of immunotherapy for cervical cancer. Rep Pract Oncol Radiother. (2018) 23:580-8. doi: 10.1016/j.rpor.2018.05.001

56. Rodríguez E, Schetters STT, van Kooyk Y. The tumour glyco-code as a novel immune checkpoint for immunotherapy. Nat Rev Immunol. (2018) 18:204-11. doi: 10.1038/nri.2018.3

Conflict of Interest Statement: The authors declare that the research was conducted in the absence of any commercial or financial relationships that could be construed as a potential conflict of interest.

Copyright (C) 2019 Sahasrabudhe, van der Horst, Spaans, Kenter, de Kroon, Bosse, van Vliet and Jordanova. This is an open-access article distributed under the terms of the Creative Commons Attribution License (CC BY). The use, distribution or reproduction in other forums is permitted, provided the original author(s) and the copyright owner(s) are credited and that the original publication in this journal is cited, in accordance with accepted academic practice. No use, distribution or reproduction is permitted which does not comply with these terms. 